\title{
Krise und Restrukturierung: Anforderungen an eine Gute-Arbeit-Strategie
}

In der arbeitspolitischen Debatte der Jahre 2008 und 2009 herrschte weitgehend Einigkeit darüber, dass wirtschaftliche Krisenund erst recht eine so große, mehrdimensionale Krise des Finanzmarktkapitalismus - noch nie die Stunde „Guter Arbeit“ waren. Krisenanalysen wiesen darauf hin, dass das Shareholder-Value-Regime zu einer fatalen unternehmenspolitischen und gesellschaftlichen Fehlsteuerung beigetragen habe, die auch arbeitspolitisch zu einer „systematische(n) Vernachlässigung der Human-Ressourcen" führe. Aus der Delegitimierung des Shareholder-Konzeptes wurde berechtigterweise die Frage nach den Chancen eines arbeitspolitischen Paradigmenwechsels ${ }^{2}$ abgeleitet. Die Nutzung einer solchen Chance erfordert allerdings eine offensive Krisenüberwindungsstrategie aufseiten der Gewerkschaften, die nicht nur auf die Verhinderung einer Beschäftigungskatastrophe orientiert, sondern auch die Verteidigung der Lohn-, Arbeits- und Sozialstandards als wichtige Handlungsfelder ins Visier nimmt. ${ }^{3}$

Aus heutiger Sicht hat sich nicht nur der Finanzmarktkapitalismus als stabiler erwiesen als erwartet, sondern es droht auch eher eine Radikalisierung von Kostensenkungsstrategien und Externalisierung der Krisenkosten auf die Gesellschaft. Was bedeutet dies für das Feld der Arbeitspolitik?

Alle Anzeichen sprechen dafür, dass in und nach der Krise, deren Ende noch nicht absehbar ist, kosteninduzierte Restrukturierungen in den Unternehmen die Arbeitsprozesse umwälzen und den Druck auf die Arbeitsstandards immens erhöhen werden. Eine Erhebung des Instituts für Arbeitsmarkt- und Berufsforschung (IAB) zu den krisenbedingten Maßnahmen von Unternehmen zeigt, dass die Mehrheit der Betriebe mehr noch als Entlassungen eine Kostensenkung durch Umstrukturierungen in Angriff nimmt. ${ }^{4}$ Der HIRES-Report einer EU-Expertengruppe „Gesundheit und Restrukturierung“ prognostiziert: „In der momentanen Wirtschaftskrise könnten die potenziellen Auswirkungen von Restrukturierung auf die Gesundheit sogar Ausmaße einer Pandemie annehmen“. Restrukturierungen führten $\mathrm{zu}$ „Unsicherheiten und Irritationen auf allen Ebenen“ und würden als „sozialer Krieg" wahrgenommen werden. Deshalb sollten „die Gewerkschaften Restrukturierungen als ein Risikopotenzial für die Gesundheit der Beschäftigten identifizieren “. ${ }^{5}$ Die Expertengruppe empfiehlt den Sozialpartnern, sie sollten „sicherstellen, dass die gesundheitlichen Folgen während eines Restrukturierungsprozesses durchgängig thematisiert werden und eine Bewertung gesundheitlicher Auswirkungen in jeder Phase des Prozesses stattfindet" ${ }^{\text {“ }}$ Dies stellt eine durchaus ambitionierte Herausforderung für eine gewerkschaftliche Intervention in Restrukturierungsprozesse dar.

Restrukturierungen sind ebenso wie Innovationsprozesse auch auf ihre ambivalenten Folgen hin zu untersuchen. Eine gewerkschaftliche Antikrisenstrategie wird den radikalisierten Kostensenkungsstrategien einen nachhaltigen innovationsorientierten Ansatz entgegensetzen. Damit wird zum einen die Auseinandersetzung mit den gescheiterten Imperativen einer Kurzfristökonomie erforderlich, da sich Innovationen erst über längere Fristen rechnen. Die Stärkung der Innovationsfähigkeit als Teil eines Krisenüberwindungskonzepts wird dabei zum einen Innovation in all ihren Dimensionen umfassen müssen: Produktinnovationen, Prozessinnovationen und soziale Innovationen. Zum anderen darf dabei aber die ,janusköpfige Wirkung" (Michael Schumann) innovativer Produktionskonzepte nicht übersehen werden. Auch innovative Modelle leis- tungspolitischer und arbeitsorganisatorischer Rationalisierung sind vielfach mit systematischen Interessenverletzungen der Beschäftigten verbunden. Entgrenzung von Leistungsanforderungen und Arbeitszeiten, Verletzung von Beschäftigteninteressen im Rahmen indirekter Personalsteuerungskonzepte sowie Segmentierung und machtpolitische Schwächung der Belegschaften gehen vielfach mit neuen Produktionskonzepten einher. Auch innovative Arbeit kann schlechte Arbeit sein.

Die WSI/PARGEMA-Betriebsrätebefragung 2008/09 bestätigt eindrucksvoll diesen ambivalenten Charakter: „Mit den Innovationen ging häufig eine Verschlechterung der Arbeitsbedingungen einher. In der Regel nahm u. a. der Arbeitsstress

Kalmbach, P./Schumann, M. (2008): Finanzkrise als Schocktherapie, in: WSI-Mitteilungen 11+12, S. 627.

2 Pickshaus, K./Urban, H.-J. (2009): Gute Arbeit in Krisenzeiten - Arbeitspolitik zwischen defensiver Krisenabwehr und offensiver Krisenüberwindung, in: WSI-Mitteilungen 6, S. 331-334.

3 Pickshaus, K./Urban, H.J. (2010): Krisenopfer Gute Arbeit? Gewerkschaftliche Arbeitspolitik in der Krise des Finanzmarkt-Kapitalismus, in: Schröder, L./Urban, H.-J. (Hrsg.): Gute Arbeit. Handlungsfelder für Betriebe, Politik und Gewerkschaften, Frankfurt, S. 39ff.

4 Heckmann, M./Kettner, A./Pausch, S./Szameitat, J./Vogler-Ludwig, K. (2009): Unternehmensbefragung im II. Quartal 2009. Wie Betriebe in der Krise Beschäftigung stützen, IAB-Kurzbericht 18, Nürnberg.

5 Kieselbach, Th. et al. (2009): Gesundheit und Restrukturierung. Innovative Ansätze und Politikempfehlungen, München und Mehring, S. 13-25. 6 Ebd. S. 86.

Klaus Pickshaus, Leiter des Funktionsbereichs Gesundheitsschutz und Arbeitsgestaltung beim Vorstand der IG Metall, Frankfurt/Main. e-mail: klaus.pickshaus@igmetall.de 
zu und der Leistungsdruck hatte sich infolge der Innovationen erhöht. Dies zeigte sich bei allen betrachteten Innovationsarten". 7

Dies spricht dafür, Fragen der Leistungsbedingungen und der Gesundheit in einer gewerkschaftlichen Krisenüberwindungsstrategie zu einem zentralen Bestandteil zu machen. Zumal alle Anzeichen darauf hindeuten, dass der Grad der Vernutzung der Arbeitskraft der abhängig Beschäftigten nicht nur in, sondern auch nach der Krise weiterhin zunehmen wird. Wie ausgeführt hat auch die HIRES-Expertengruppe bei Restrukturierungen eine Fokussierung auf Folgen für die Gesundheit empfohlen. Eine solche Schwerpunktsetzung entspricht dem hohen Stellenwert, den diese Themen für die Beschäftigten trotz des Krisendrucks besitzen. Befragungen belegen das nach wie vor hohe Anspruchsniveau der Beschäftigten, ihre Erwartungen an eine sichere und gute Arbeit. In der Beschäftigtenbefragung der IG Metall im Frühsommer 2009 (470.000 Befragte) erhalten „Beschäftigungssicherheit“ und danach „Arbeit, die nicht krank macht" die höchsten Zustimmungswerte. Die Befunde der repräsentativen jährlichen Erhebung des DGB-Index Gute Arbeit bestätigen dies. Aus Sicht der Beschäftigten ist gute Arbeit an ein hohes Maß an Beschäftigungs- und Einkommenssicherheit, an Schutz vor zu hohen körperlichen und psychischen Belastungen sowie an die Existenz ausreichender Ressourcen gebunden, die Entwicklungsmöglichkeiten und das Wohlbefinden und die Zufriedenheit in der Arbeit gewährleisten. Durch das Instrument des DGB-Indexes Gute Arbeit wird die Bewertung der Qualität der Arbeit durch die Beschäftigten selbst, jenseits von quasi objektiven Experteneinschätzungen, in den Mittelpunkt gerückt.

Alle empirischen Befunde sprechen dafür, dass in der gegenwärtigen Krise nicht nur Grenzen einer Ökonomie der Maßlosigkeit, sondern auch Anzeichen einer Überforderung der Menschen durch überlange Arbeitszeiten, Verdichtung der Arbeit und Grenzen von Motivation und Engagement sichtbar werden. Nach der WSI-Betriebsrätebefragung 2008/2009 geben $79 \%$ der Betriebsräte an, dass die psychischen Belastungen in den letzten drei Jahren zugenommen haben und 26\% nehmen auch eine Zunahme körperlicher Belastungen wahr. 84 \% der Betriebsräte konstatieren, dass die Belegschaften unter ständig hohem Zeit- und Leistungsdruck stehen.

Eine weitere Zuspitzung der Belastungen und Zumutungen droht den „seidenen Faden der Gesundheit" zum Reißen zu bringen, der in vielen Befunden schon als „angespannt“ geschildert wird. Das kann aber auch bedeuten: Der Kampf um Gesundheit in der Arbeit könnte zu einem zentralen Konfliktfeld werden, in dem erhebliche Widerstandspotenziale gegen die Zumutungen einer fortgesetzten Shareholder-Ökonomie liegen. Möglicherweise schaffen die Permanenz der Bedrohungen der Gesundheit und die hohe Wertschätzung ihrer Bewahrung unter den Beschäftigten trotz schwieriger Krisenbedingungen neue Aktivierungsansätze.

Ein qualitativ ausgerichtetes Antikrisenkonzept sollte deshalb über die unmittelbare Verhinderung von Entlassungen hinaus erweitert werden und auch die Abwehr von Einkommenskürzungen und verschlechterten Arbeitsbedingungen umfassen. Bei der Abwehr schlechter Arbeit können Themen wie Schutz der Gesundheit und Erhalt der Arbeitsfähigkeit eine zusätzliche Mobilisierungskraft entfalten, die den Handlungsfeldern Arbeitszeit- und Leistungspolitik sowie Arbeitsgestaltung eine Zuspitzung verleihen.

Die Stärkung und Mobilisierung solcher Widerstandspotenziale zum Erhalt von Gesundheit und Arbeitsfähigkeit haben mehrfach positive Wirkungen: Sie befördern die Artikulation der autonomen Interessen der Beschäftigten und damit die Gegenmachtfähigkeit. Zugleich stärken sie die gesundheitsförderlichen Potenzen der Beschäftigten. Denn eigenes Engagement zum Erhalt der Gesundheit, Widerstand gegen die Zumutungen der Ökonomie und selbstaktives Einwirken auf die unmittelbaren Arbeitsumweltbedingungen tragen ihrerseits zur Ressourcenstärkung bei den Betroffenen bei, mit denen sie Belastungen besser abfedern können. Stärkung von Kollegialität und Solidarität, Konfliktfähigkeit und Widerstandskraft sind zugleich Komponenten der Entfaltung gewerkschaftlicher Handlungsmacht und der Förderung individueller Ressourcen zur besseren Bewältigung arbeitsbedingter Fehlbelastungen. Die Arbeitspsychologie unterstützt aus salutogener Perspektive die Einschätzung, dass Angst und Anpassung in der Krise auch aus Gesundheitsgründen die unterlegene Reaktionsform darstellen: „Natürlich ist Aufbegehren gesünder.
Menschen, die sich stark engagieren, über Streiks, Betriebsbesetzungen, kollektiven Widerstand, leiden selbst unter Stress seltener an Burn-out und Erschöpfung. Gerade die Gewerkschaften versuchen ja auch, eine betriebliche Solidarität aufzubauen“" ${ }^{8}$

Aufbegehren und Widerstand, und vor allem die kollektive und solidarische Veränderung der eigenen Arbeitsbedingungen brauchen entsprechende Einwirkungsmöglichkeiten und Rechte, brauchen demokratische Einflussmöglichkeiten im Betrieb. Damit wird Demokratie in der Arbeit als essenzielle Voraussetzung für eine erfolgreiche Krisenüberwindungsstrategie deutlich. Gute Arbeit muss demokratische Arbeit sein. ${ }^{9}$

Auf betrieblicher Ebene wird es zum einen um erweiterte Mitbestimmungsund Autonomierechte am Arbeitsplatz und im Betrieb gehen. Damit wird auch die leistungspolitische Erschöpfung der Beschäftigten in der Krise zu thematisieren sein, die ihrerseits die Wahrnehmung schon bestehender Mitbestimmungsrechte zu blockieren droht. Zum anderen wird die Politik des unternehmens- und wirtschaftspolitischen „Weiter so“ durch eigene strukturpolitische - produktorientierte, soziale und ökologische - Umbauprogramme infrage zu stellen sein. Der von der IG Metall vorgeschlagene Beteiligungsfonds („public equity“) zur Unternehmenssicherung könnte einen Weg der öffentlich finanzierten und kontrollierten Investitionssteuerung eröffnen, in dem auch die arbeitspolitischen Entwicklungspfade beeinflusst werden können. ${ }^{10}$ Dörrel Holst ergänzen die inklusiven Möglichkeiten: „Solche Ansätze könnten zudem mit einer arbeitspolitischen Perspektive verbunden werden, die die workforce von

7 WSI-Betriebsrätebefragung 2008/09 zu Innovationsfähigkeit, Arbeitsbedingungen und Gesundheit im Betrieb, Informationsblatt der HansBöckler-Stiftung 2009; Ziegler, A. (2010): Welche Auswirkungen haben betriebliche Innovationen auf die Beschäftigten?, in: WSI-Mitteilungen 2, S. 108, Abb. 4.

8 Richter, P. (2009): Frankfurter Rundschau vom 29.4.; Richter, P. (2009): Interview, in: AiBplus 4, S. 13.

9 Pickshaus, K./Urban, H.-J. (2009): Gute Arbeit als Strategie - Perspektiven gewerkschaftlicher Arbeitspolitik, in: Schröder, L./Urban, H.J. (Hrsg.) (2009): Gute Arbeit. Handlungsfelder für Betriebe, Politik und Gewerkschaften, Frankfurt, S. $108 \mathrm{ff}$.

10 IG Metall Vorstand (2009): Aktiv aus der Krise Gemeinsam für ein Gutes Leben. Aktionsplan der IG Metall, Frankfurt/M. (www.igmetall.de). 
Wirtschaftsorganisationen als Ganzes in den Blick nimmt “. ${ }^{11}$ Ohne eine Stärkung wirtschaftsdemokratischer Einflussmöglichkeiten ist jedenfalls eine grundlegende Abkehr vom finanzmarktdominierten Typus der Unternehmens- und Wirtschaftspolitik schwer vorstellbar.

Nach fast zwei Jahren Krise kann man bilanzieren, dass eine selbstkritische Aufarbeitung der fatalen unternehmenspolitischen und gesellschaftlichen Fehlsteuerung nach wie vor aussteht. Kuhlmann resümiert zutreffend: „Es gibt bisher keine Hinweise, dass es zu einer Umorientierung bei den arbeitspolitischen Leitbildern kommt" ${ }^{12}$ Man muss vielmehr konstatieren, dass die Gefahr besteht, dass mit den gescheiterten Rezepten die Zumutungen für Beschäftigte radikalisiert und die Arbeitsbedingungen weiter verschlechtert werden. Auch des- halb wird das Thema der Demokratie in der Arbeit machtpolitisch zu einer Schlüsselfrage. Kuhlmann bestimmt die Aufgabe der Arbeitspolitik nach der Krise wie folgt: "Ausgehend von Maßstäben, guter Arbeit' geht es darum, eine Auseinandersetzung über arbeitspolitische Leitbilder zu führen, für die sich vor dem Hintergrund der mittlerweile wiederbelebten Debatte über Wirtschaftsdemokratie und Unternehmensmitbestimmung neue Bezugspunkte ergeben". 13

Gerade in zugespitzten Krisen- und Konfliktphasen erweisen sich demokratische Einwirkungsmöglichkeiten als unverzichtbare Voraussetzungen humaner Arbeitsgestaltung. Nicht nur, weil die Ermöglichung und Förderung von Eigeninitiativen und Partizipation Essentials für die Herstellung „salutogener Settings“ im Betrieb darstellen. Sondern auch, weil sich nur über die Mobilisierung von kollektiver Selbsthilfe, arbeitspolitischer Verhandlungsmacht und realen Einwirkungen auf die Umbrüche in der Arbeitswelt das Mögliche und Notwendige realisieren lässt: Eine Arbeitspolitik, die gute Arbeit nicht der Krise des Finanzkapitalismus opfert, sondern als Leitbild einer weitreichenden Neuorganisation der Arbeit aufwertet.

11 Dörre, K./Holst, H. (2009): Nach dem Shareholder Value? Kapitalmarktorientierte Unternehmenssteuerung in der Krise, in: WSI-Mitteilungen $11+12$, S. 673 .

12 Kuhlmann, M. (2009): Perspektiven der Arbeitspolitik nach der Krise: Entwicklungslinien und Handlungsbedingungen, in: WSI-Mitteilungen $11+12$, S. 680 .

13 Ebd. S. 681. 